\title{
THE COHOMOLOGY GROUPS OF A FIBRE SPACE WITH FIBRE A SPACE OF TYPE $\kappa(\pi, n)$
}

\author{
W. H. COCKCROFT ${ }^{1}$
}

1. Introduction. The cohomology groups $H^{q}(\pi, n ; G)$ of a space of type $\pi(\pi, n)$, i.e. with one nonvanishing homotopy group, $\pi$, in dimension $n$, have been fully determined by $\mathrm{H}$. Cartan, S. Eilenberg, and Saunders MacLane. ${ }^{2}$ If however one turns to a space with two nonvanishing homotopy groups, say $\pi_{m}, \pi_{n}, m>n$, the relevant theo$\mathrm{rem}^{3}$ of Eilenberg-MacLane gives the cohomology groups only as far as dimension $m$. Now such a space is well known ${ }^{4}$ to be of the same homotopy type as a fibre space with fibre of type $\mathscr{K}\left(\pi_{m}, m\right)$ and base of type $\Re\left(\pi_{n}, n\right)$. My object here is to show that using this fact, standard spectral sequence arguments give information over a field of coefficients, at least as far as dimension $m+2$, in case $m>n>1$, without undue complication.

Since such a fibre space is a particular example of one in which only the fibre is restricted to be of type $\mathcal{K}(\pi, n)$, I shall prove my results for these more general spaces. Such spaces have of course occurred often in the literature in recent years. Indeed associated with any space $X$ there is a sequence $\{(X, n)\}$ of spaces, with $(X, n)$ a fibre space with fibre of type $\mathfrak{K}\left(\pi_{n}(X), n\right)$ and base $(X, n-1) .^{5}$

I am indebted to Professor E. Spanier for many helpful conversations on this and allied topics, and to Professor Saunders MacLane for his comments on the results.

2. Statement of theorems. Let $E$ denote a fibre space with fibre $F$ a space of type $\mathfrak{K}(\pi, n), n \geqq 2$, i.e. $\pi_{i}(F)=0, i \neq n, \pi_{n}(F)=\pi$. Let the base space $B$ of $E$ be arcwise connected and simply connected. All the spaces with which I shall be concerned here will be assumed to be such that their cohomology groups are finitely generated. Let $k^{n+1}$ $\in H^{n+1}(B ; \pi)$ denote the characteristic cohomology class of $E$, de-

Presented to the Society, September 2, 1955; received by the editors December 17, 1955.

${ }^{1}$ Part of this work was done while the author was supported by the Carnegie Trust for the Universities of Scotland, and by the National Science Foundation, Grant No. 1008.

${ }^{2}$ Cf. $[1 ; 3 ; 4]$. Numbers in square brackets refer to the list of references at the end of this note.

3 [6, Theorem IV.]

4 Cf. [8, Chap. III, §2].

${ }^{5}$ See footnote 4 . 
fined to be minus the transgression of the basic cohomology class of the fibre, i.e. $k^{n+1}=-\tau_{n+1} u^{n}$, where $\tau_{n+1}: H^{n}(F ; \pi) \rightarrow H^{n+1}(B ; \pi)$ denotes the transgression homomorphism, ${ }^{6}$ and

$$
u^{n} \in H^{n}(F ; \pi) \approx \operatorname{Hom}(\pi, \pi)
$$

is the cohomology class corresponding to the identity isomorphism of $\pi$ with itself. Finally let $A$ be any field of coefficients, and let $\operatorname{Hom}^{*}(\pi, A)$ denote the subgroup of $\operatorname{Hom}(\pi, A)$ which consists of those homomorphisms $\rho: \pi \rightarrow A$ such that $\rho^{*} k^{n+1}=0$, where

$$
\rho^{*}: H^{n+1}(B ; \pi) \rightarrow H^{n+1}(B ; A)
$$

will always denote the homomorphism induced by such a $\rho$.

THEOREM 1.

(i) $H^{i}(E ; A) \approx H^{i}(B ; A) 0 \leqq i \leqq n-1$;

(ii) $H^{n}(E ; A)$ is a group extension of $\operatorname{Hom}^{*}(\pi, A)$ by $H^{n}(B ; A)$.

Let

$$
\delta_{\theta}: H^{i}(B ; \pi) \rightarrow H^{i+1}(B ; A)
$$

be the coboundary homomorphism associated with an element $\theta: 0 \rightarrow A \rightarrow G \rightarrow \pi \rightarrow 0$ of the group, Ext Abel $(\pi, A)$, of Abelian group extensions of $\pi$ by $A$. Let

$$
\operatorname{Ext~} \operatorname{Abel}^{*}(\pi, A)
$$

denote the subgroup of Ext Abel $(\pi, A)$ which consists of those extensions $\theta$ such that $\delta_{\theta} k^{n+1}=0$, and let $\mathfrak{S}^{n+1}(B ; A)$ denote the factor group of $H^{n+1}(B ; A)$ by the subgroup of elements of the form $\rho^{*} k^{n+1}$, for all $\rho \in \operatorname{Hom}(\pi, A)$.

Theorem $2 .{ }^{7} H^{n+1}(E ; A)$ is a group extension of Ext Abel* $(\pi ; A)$ by $\mathfrak{S}^{n+1}(B ; A)$.

Now let $n>2$. Following Eilenberg-MacLane ${ }^{8}$ let $S q_{i}^{2}: H^{q}(K ; \pi)$ $\rightarrow H^{q+2}(K ; A), K=B, F$, denote the "Steenrod square" associated with any trace function $t: \pi \rightarrow A$, that is, with any homomorphism $t \in \operatorname{Hom}(\pi, A)$ such that $t(x)-t(-x)=0$ for all $x \in \pi$. Let $T^{*}(\pi, A)$ denote the subgroup of $\operatorname{Hom}(\pi, A)$ of all such trace functions which satisfy the condition $S q_{l}^{2} k^{n+1}=0$. Let $\mathfrak{Q}^{n+2}(B ; A)$ denote the factor group of $H^{n+2}(B ; A)$ by the subgroup of elements of the form $\delta_{\theta} k^{n+1}$, for all $\theta \in \operatorname{Ext}$ Abel $(\pi, A)$. Finally let $P$ denote the subgroup of elements $\sum b^{2} \otimes \rho \in H^{2}(B ; A) \otimes \operatorname{Hom}(\pi, A)$ such that $\sum \boldsymbol{b}^{2} \cup \rho^{*} k^{n+1}=0$.

- Defined, e.g. as in [7, Chap. II, §9].

7 This corrects the result as first announced in Bull. Amer. Math. Soc. Abstract 61-6-828, The cohomology groups of certain fibre spaces.

${ }^{8}$ See $[2]$. 
TheOREM $3 .^{7} H^{n+2}(E ; A)$ has a composition series

$$
H^{n+2}(E ; A) \supset D^{* 1, n+1} \supset D^{* n+2,0} \supset 0
$$

where

$$
\begin{aligned}
H^{n+2}(E ; A) / D^{* 1, n+1} & \approx T^{*}(\pi ; A), \\
D^{* 1, n+1} / D^{* n+2,0} & \approx P \\
D^{* n+2,0} & \approx \mathfrak{S}^{n+2}(B ; A) .
\end{aligned}
$$

As indicated in Theorem 3, the notation used in the spectral sequence arguments will be precisely that of [7]. In case $B$ is a CW complex it is well known that the definition of $\boldsymbol{k}^{n+1}$ used above coincides with the definition of the characteristic class in terms of obstruction theory. ${ }^{9}$ Thus if $B$ has a single nonvanishing homotopy group in dimension less than $n$, the Eilenberg-MacLane result is recovered, at least for $\mathrm{CW}$ complexes.

3. Proof of Theorem 1. Since $H^{1}(B ; A)=0$ and $H^{j}(F ; A)=0$, $0<j<n$, we can deduce ${ }^{10}$ from the cohomology spectral sequence $\left(E_{r}^{*}, d_{r}\right)$ associated with $E$ the existence of the exact sequence

$$
\begin{gathered}
0 \rightarrow H^{1}(B ; A) \rightarrow H^{1}(E ; A) \rightarrow H^{1}(F ; A) \stackrel{d_{2}}{\rightarrow} H^{2}(B ; A) \rightarrow \cdots \\
\cdots \rightarrow H^{n}(F ; A) \stackrel{d_{n+1}}{\longrightarrow} H^{n+1}(B ; A) \rightarrow H^{n+1}(E ; A) \rightarrow H^{n+1}(F ; A),
\end{gathered}
$$

where $d_{i}$ is the transgression homomorphism. Part (i) of Theorem 1 follows immediately from the exactness of this sequence, since $H^{j}(F ; A)=0,0<j<n$.

Also from the exactness of the sequence, it follows that $H^{n}(E ; A)$ is a group extension of $d_{n+1}^{-1}(0)$ by $H^{n}(B ; A)$, so that part (ii) of the theorem requires simply the calculation of the kernel of the transgression $d_{n+1}$. Since $F$ is of type $\Re(\pi, n), H^{n}(F ; A)=H^{n}(\pi, n ; A)$, and $H^{n}(\pi, n ; A) \approx \operatorname{Hom}(\pi, A)$, this last isomorphism being given ${ }^{11}$ by the correspondence $\rho^{*} u^{n} \leftrightarrow \rho$, where $\rho \in \operatorname{Hom}(\pi, A), \rho^{*}$ is induced by $\rho$, and, as above, $\boldsymbol{u}^{n}$ is the basic cohomology class in $H^{n}(\pi, n ; A)$. Thus any element of $H^{n}(F ; A)$ will be written $\rho^{*} u^{n}$ for some $\rho \in \operatorname{Hom}(\pi, A)$. If, as in $\S 2, \tau_{n+1}$ is the transgression when $\pi$ is the group of coefficients, we have $d_{n+1} \rho^{*} u^{n}=\rho^{*} \tau_{n+1} u^{n}$, since $\rho^{*}$ is essentially a change of coefficients and thus commutes with the transgression. Hence

- See [9].

${ }^{10}$ Cf. [7, Chap. III, Prop. 5, Remark 1].

${ }^{11} \mathrm{Cf} .[5, \S 8]$. 
$d_{n+1} \rho^{*} u^{n}=0$ if and only if $\rho^{*} k^{n+1}=0$, i.e. under the correspondence $\rho^{*} u^{n} \leftrightarrow \rho$, the subgroup $d_{n+1}^{-1}(0)$ of $H^{n}(F ; A)$ is isomorphic to the subgroup Hom* $(\pi, A)$ of Hom $(\pi, A)$ which consists of those homomorphisms $\rho: \pi \rightarrow A$ such that $\rho^{*} k^{n+1}=0$. This proves part (ii) of Theorem 1.

4. Proof of Theorem 2. From the exactness of the sequence of $\$ 3$, $H^{n+1}(E ; A)$ is a group extension of its image in $H^{n+1}(F ; A)$, by $H^{n+1}(B ; A) / d_{n+1} H^{n}(F ; A)$. Since $d_{n+1} \rho^{*} u^{n}=-\rho^{*} k^{n+1}, d_{n+1} H^{n}(F ; A)$ is the subgroup of $H^{n+1}(B ; A)$ which consists of elements of the form $\rho^{*} k^{n+1}$ for all $\rho \in \operatorname{Hom}(\pi, A)$, as required. Thus it remains to calculate the image of $H^{n+1}(E ; A)$ in $H^{n+1}(F ; A)$. Here we are beyond the range of the exact sequence and required to use the spectral sequence arguments from which the exact sequence was obtained in the first place. Firstly, we have a composition series

$$
H^{n+1}(E ; A)=D^{* 0, n+1} \supset D^{* 1, n} \supset \cdots \supset D^{* n+2,-1}=0,
$$

where $D^{* p, q} / D^{* p+1, q-1}=E_{\infty}^{* p, q}$. Since $A$ is a field and the cohomology groups are finitely generated,

$$
E_{2}^{*}{ }^{p, q}=H^{p}\left(B ; H^{q}(F ; A)\right) \approx H^{p}(B ; A) \otimes H^{q}(F ; A) .
$$

Hence $E_{2}^{* p, q}=0$ if $p+q=n+1, p \neq 0, n+1$, in virtue of the hypotheses on $B$ and $F$. Thus $E_{\infty}^{* p, q}=0$ if $p+q=n+1, p \neq 0, n+1$, and the above composition series for $H^{n+1}(E ; A)$ reduces to

$$
H^{n+1}(E ; A) \supset D^{* 1, n} \supset 0
$$

where $H^{n+1}(E ; A) / D^{* 1, n}=E_{\infty}^{* 0, n+1}$, and $D^{* 1, n}=E_{\infty}^{* n+1,0}$. The term $D^{* 1, n}$ is, of course, precisely the factor group $H^{n+1}(B ; A) / d_{n+1} H^{n}(F ; A)$ as obtained in the exact sequence of $\S 3$, and I omit the details of its precise calculation. We are left therefore with $E_{\infty}^{* 0, n+1}$ (which is the image of $H^{n+1}(E ; A)$ in $\left.H^{n+1}(F ; A)\right)$ to calculate. We have $H^{n+1}(F ; A)$ $=H^{n+1}(\pi, n ; A) \approx$ Ext Abel $(\pi, A)$, where this last isomorphism is given $^{11}$ by the correspondence $\Delta_{\theta} \mathbf{u}^{n} \leftrightarrow \theta$, for all coboundary homomorphisms $\Delta_{\theta}: H^{n}(F ; \pi) \rightarrow H^{n+1}(F ; A)$ associated with the elements $\theta \in$ Ext Abel $(\pi, A)$. Since the basic class $u^{n} \in H^{n}(F ; \pi)$ is transgressive, it follows ${ }^{12}$ that $\Delta_{\theta} u^{n}$ is transgressive, and since any element of $H^{n+1}(F ; A)$ can be written in the form $\Delta_{\theta} u^{n}$, for some $\theta$, all the elements of $H^{n+1}(F ; A)$ are transgressive. Thus

$$
H^{n+1}(F ; A)=E_{2}^{* 0, n+1}=\cdots=E_{n+2}^{* 0, n+1} \supset E_{n+3}^{* 0, n+1}=\cdots=E_{\infty}^{* 0, n+1} \text {, }
$$

12 This follows from the fact that $\Delta_{\theta}$ commutes with transgression, which is a consequence of a parallel argument to that given by Serre [7, Chap. II, \$9, (c)], for the commutativity of the Steenrod square. 
and $E_{\infty}^{* 0, n+1}$ is the kernel of the transgression homomorphism $d_{n+2}$ : $E_{n+2}^{* 0, n+1} \rightarrow E_{n+2}^{* n+2,0}$. But ${ }^{13} d_{n+2} \Delta_{\theta} u^{n}=\delta_{\theta} \tau_{n+1} u^{n}$, where $\delta_{\theta}: H^{n+1}(B ; \pi)$ $\rightarrow H^{n+2}(B ; A)$ is the coboundary homomorphism also associated with $\theta \in$ Ext Abel $(\pi, A)$. Thus $d_{n+2} \Delta_{\theta} u^{n}=0$ if and only if $\delta_{\theta} \tau_{n+1} u^{n}$ $\left(=-\delta_{\theta} k^{n+1}\right)=0$, i.e. under the correspondence $\Delta_{\theta} u^{n} \leftrightarrow \theta$ the subgroup $E_{\infty}^{* 0, n+1}$ of $H^{n+1}(F ; A)$ is isomorphic to the subgroup Ext $\mathrm{Abel}^{*}(\pi, A)$ of Ext Abel $(\pi, A)$, which consists of those $\theta \in \operatorname{Ext}$ Abel $(\pi, A)$ such that $\delta_{\theta} k^{n+1}=0$, and the theorem is proved.

5. Proof of Theorem 3. The cohomology spectral sequence associated with $E$ yields the composition series for $H^{n+2}(E ; A)$ :

$$
\begin{aligned}
B^{n+2}(E ; A) & =D^{* 0, n+2} \supset D^{* 1, n+1} \supset D^{* 2, n} \supset \cdots \supset D^{* n+2,0} \\
& \supset D^{* n+3,-1}=0,
\end{aligned}
$$

where $D^{* p, q} / D^{* p+1, q-1}=E_{\infty}^{* p, q}$. As in $\$ 4, E_{2}^{* p, q}=H^{p}\left(B ; H^{q}(F ; A)\right)$ $\approx H^{p}(B ; A) \otimes H^{q}(F ; A)$, and hence $E_{2}^{* p, q}=0$ if $p+q=n+2, p \neq 0,2$, $n+2$, because of the conditions on $B$ and $F$. Thus $E_{\infty}^{*}{ }^{p, q}=0$ if $p+q$ $=n+2, p \neq 0,2, n+2$, and the composition series reduces to

$$
B^{n+2}(E ; A) \supset D^{* 1, n+1} \supset D^{* n+2,0} \supset 0,
$$

where

$$
\begin{gathered}
H^{n+2}(E, A) / D^{* 1, n+1}=E_{\infty}^{* 0, n+2}, \\
D^{* 1, n+1} / D^{* n+2,0}=E_{\infty}^{* 2, n},
\end{gathered}
$$

and

$$
D^{* n+2,0}=E_{\infty}^{* n+2,0}
$$

To prove the desired result we therefore calculate these three $E_{\infty}^{*}$ terms.

(a) $E_{\infty}^{* n+2,0}$. All the elements of $E_{r}^{* n+2,0}$ are cocycles for $d_{r}$, if $r>1$. Nontrivial coboundaries occur only when $r=n+2$. Thus

$$
\begin{aligned}
H^{n+2}(B ; A) & =E_{2}^{* n+2,0}=E_{3}^{* n+2,0}=\cdots=E_{n+2}^{* n+2,0} \rightarrow E_{n+3}^{* n+2,0} \\
& =\cdots=E_{\infty}^{* n+2,0},
\end{aligned}
$$

and

$$
E_{\infty}^{* n+2,0} \approx H^{n+2}(B ; A) / d_{n+2} E_{n+2}^{* 0, n+1}=H^{n+2}(B ; A) / d_{n+2} H^{n+1}(F ; A) .
$$

But $d_{n+2} H^{n+1}(F ; A)$ is the subgroup of elements of $H^{n+2}(B ; A)$ of the form $d_{n+2} \Delta_{\theta} u^{n}=\delta_{\theta} \tau_{n+1} u^{n}=-\delta_{\theta} k^{n+1}$, for all $\theta \in \operatorname{Ext}$ Abel $(\pi, A)$. Thus $E_{\infty}^{* n+2,0} \approx \mathfrak{S}^{n+2}(B ; A)$ as required.

is See footnote 12 . 
(b) $E_{\infty}^{* 2, n}$. Again the hypotheses imply that

$$
E_{2}^{* 2, n}=E_{3}^{* 2, n}=\cdots=E_{n+1}^{* 2, n} \supset E_{n+2}^{* 2, n}=\cdots=E_{\infty}^{* 2, n},
$$

so that $E_{\infty}^{* 2, n}$ is the kernel of $d_{n+1}: E_{n+1}^{* 2, n} \rightarrow E_{n+1}^{* n+3,0}$. Moreover $E_{2}^{* 2, n}$ $\approx H^{2}(B ; A) \otimes H^{n}(F ; A)$ so that any element of $E_{2}^{* 2, n}$ can be written in the form of a finite sum $\sum b^{2} \otimes \rho^{*} u^{n}$, for $b^{2} \in H^{2}(B ; A), \rho \in \operatorname{Hom}(\pi$, $A$ ) and $\boldsymbol{u}^{n}$ the fundamental class in $H^{n}(F ; \pi)$. Now

$$
\begin{aligned}
d_{n+1}\left(\sum b^{2} \otimes \rho^{*} u^{n}\right) & =d_{n+1}\left(\sum\left(b^{2} \otimes 1\right)\left(1 \otimes \rho^{*} u^{n}\right)\right) \\
& =\sum\left(b^{2} \otimes 1\right)\left(1 \otimes d_{n+1} \rho^{*} u^{n}\right) \\
& =-\sum\left(b^{2} \otimes 1\right)\left(1 \otimes \rho^{*} k^{n+1}\right) \\
& =-\sum b^{2} \otimes \rho^{*} k^{n+1} \\
& =-\sum b^{2} \cup \rho^{*} k^{n+1} .
\end{aligned}
$$

Thus, $E_{\infty}^{* 2, n}$ is the subgroup of $H^{2}(B ; A) \otimes H^{n}(F ; A)$ which consists of elements $\sum b^{2} \otimes \rho^{*} u^{n}$ such that $\sum b^{2} \cup \rho^{*} k^{n+1}=0$ for all $b^{2} \in H^{2}(B ; A)$ and $\rho \in \operatorname{Hom}(\pi, A)$, i.e. $E_{\infty}^{*}{ }^{2, n}$ is isomorphic to the subgroup of $H^{2}(B ; A) \otimes \operatorname{Hom}(\pi, A)$ consisting of those elements $\sum b^{2} \otimes \rho$ such that $\sum b^{2} \cup \rho^{*} k^{n+1}=0$.

(c) $E_{\infty}^{*}{ }^{0, n+2}$. Since $F$ is of type $\varkappa(\pi, n), H^{n+2}(F ; A)=H^{n+2}(\pi, n ; A)$ which is isomorphic to the group $T(\pi, A)$ of all trace functions from $\pi$ to $A$, the isomorphism being given ${ }^{14}$ by the correspondence $S q_{\imath}^{2} u^{n} \leftrightarrow t$ for all $t \in T(\pi, A)$. Since the basic class $u^{n} \in H^{n}(F ; \pi)$ is transgressive, its Steenrod square is transgressive ${ }^{15}$ and all the elements of $H^{n+2}(F ; A)$ are transgressive. Thus

$$
H^{n+2}(F ; A)=E_{2}^{* 0, n+2}=\cdots=E_{n+3}^{* 0, n+2} \supset E_{n+4}^{* 0, n+2}=\cdots=E_{\infty}^{* 0, n+2} \text {, }
$$

and $E_{\infty}^{* 0, n+2}$ is the kernel of the transgression $d_{n+3}: E_{n+3}^{* 0, n+2} \rightarrow E_{n+3}^{* n+3,0}$. But ${ }^{15} d_{n+3} S q_{t}^{2} u^{n}=S q_{t}^{2} \tau_{n+1} u^{n}=-S q_{l}^{2} k^{n+1}$, so that $E_{\infty}^{*}{ }^{0, n+2}$ is the subgroup of $H^{n+2}(F ; A)$ which consists of all elements $S q_{t}^{2} u^{n}$ such that $S q_{t}^{2} k^{n+1}=0$, i.e. the subgroup which is isomorphic under the correspondence $S q_{t}^{2} u^{n} \leftrightarrow t$ to the subgroup $T^{*}(\pi, A)$ of $T(\pi, A)$ which consists of those trace functions $t$ such that $S q_{t}^{2} k^{n+1}=0$. This completes that proof of Theorem 3 .

\section{BIBLIOGRAPHY}

1. H. Cartan, Sur les groupes d'Eilenberg-MacLane $H(\pi, n)$, I, II, Proc. Nat. Acad. Sci. U. S. A. vol. 40 (1954) pp. 467-471, 704-707.

2. Samuel Eilenberg, Homotopy groups and algebraic homology theories, Proceedings of the International Congress of Mathematicians vol. 2 (1950) pp. 350-353.

${ }^{14}$ Cf. $[5,817]$.

${ }^{16}$ See [7, Chap. II, $\left.\$ 9,(c)\right]$. 
3. Samuel Eilenberg and Saunders MacLane, On the groups $H(\pi, n)$ I, Ann. of Math. vol. 58 (1953) pp. 55-106.

4. - On the groups $H(\pi, n)$ II, Ann. of Math. vol. 60 (1954) pp. 49-139.

5. - On the groups $H(\pi, n)$ III, Ann. of Math. vol. 60 (1954) pp. 513-557.

6. - Relations between homology and homotopy groups of spaces II, Ann. of Math. vol. 51 (1950) pp. 514-533.

7. J.-P. Serre, Homologie singulière des espaces fibrés. Applications, Ann. of Math. vol. 54 (1951) pp. 425-505.

8. - Groupes d'homotopie et classes de groupes abelians, Ann. of Math. vol. 58 (1953) pp. 258-294.

9. G. Whitehead, On the characteristic cohomology class of a fibre bundle, Bull. Amer. Math. Soc. Abstract 55-5-261.

The University of Chicago and

The University of AbERDEen

\section{TEST SPACES FOR DIMENSION $n$}

\section{BYRON H. MCCANDLESS}

In 1935 Hurewicz published his celebrated theorem characteriaing the dimension of separable metric spaces by means of mappings into the $n$-sphere $S^{n}$. In this note we shall be concerned with the "dimension testing" property which $S^{n}$ displays in Hurewicz's theorem. That is, we shall try to determine the properties of a space $Y^{n}$ which enable it to characterize the dimension of separable metric spaces. Let us agree from the outset that only nonempty separable metric spaces will be considered in this problem. To make the above more precise, let $n$ be a non-negative integer and $Y^{n}$ a separable metric space. In case $Y^{n}$ satisfies the statement:

A separable metric space $X$ has dimension $\leqq n$ if, and only if, given a closed subset $C$ of $X$ and mapping $f: C \rightarrow Y^{n}$ there is an extension of $f$ over $X$, we shall call $Y^{n}$ a test space for dimension $n$. Our problem, then, is to characterize these spaces $Y^{n}$.

A special case of the problem seems to be general knowledge, although I have seen no mention of it in the literature. It is known, namely, that an arcwise connected space $Y^{n}$ tests the dimension of locally finite $k$-polytopes $(1 \leqq k \leqq n)$ if and only if $\pi_{i}\left(Y^{n}\right)=0, i<n$,

Presented to the Society, February 25, 1956; received by the editors November 14, 1955 and, in revised form, January 4, 1956.

1 Supported in part by the Research Council of Rutgers University. 